\title{
A spatial model of Wild Poliovirus Type 1 in Kano State, Nigeria: calibration and assessment of elimination probability
}

\author{
Kevin A. McCarthy ${ }^{1 *}$, Guillaume Chabot-Couture ${ }^{1}$ and Faisal Shuaib ${ }^{2}$
}

\begin{abstract}
Background: Since the launch of the Global Polio Eradication Initiative, all but three countries (Nigeria, Pakistan, and Afghanistan) have apparently interrupted all wild poliovirus (WPV) transmission, and only one of three wild serotypes has been reported globally since 2012. Countrywide supplemental immunization campaigns in Nigeria produced dramatic reduction in WPV Type 1 paralysis cases since 2010 compared to the 2000's, and WPV1 has not been observed in Nigeria since July 24, 2014. This article presents the development and calibration of a spatial metapopulation model of wild poliovirus Type 1 transmission in Kano State, Nigeria, which was the location of the most recent WPV1 case and 5 out of 6 of the reported WPV1 paralytic cases in Nigeria in 2014.

Methods: The model is calibrated to data on the case counts and age at onset of paralysis from 2003-2009. The features of the data drive model development from a simple susceptible-exposed-infective-recovered (SEIR) model to a spatial metapopulation model featuring seasonal forcing and age-dependent transmission. The calibrated parameter space is then resampled, projected forward, and compared to more recent case counts to estimate the probability that Type 1 poliovirus has been eliminated in Kano state.
\end{abstract}

Results: The model indicates a $91 \%$ probability that Type 1 poliovirus has been eliminated from Kano state as of October 2015. This probability rises to $>99 \%$ if no WPV1 paralysis cases are detected for another year. The other states in Nigeria have experienced even longer case-free periods (the only other state with a WPV1 case was Yobe, on April 19, 2014), and Nigeria is the last remaining country in Africa to experience endemic WPV1 transmission, so these results can be interpreted as an upper bound on the probability that WPV1 transmission is currently interrupted continent-wide.

Conclusions: While the results indicate optimism that WPV1 transmission has been interrupted in Kano state, the model also assumes that frequent SIAs with high coverage continue to take place in Kano state through the end of the certification period. We conclude that though WPV1 appears to be on the brink of continent-wide elimination (WHO officially removed Nigeria from the list of polio-endemic countries on September 25, 2015), it is important for the polio program to maintain vigilance in surveillance and vaccination activities to prevent WPV1 resurgence through the WHO's 3-year eradication certification period.

Abbreviations: AFP, Acute flaccid paralysis; bOPV, Bivalent oral polio vaccine; GPEl, Global polio eradication initiative; IMIS, Incremental mixture importance sampling; IPV, Inactivated polio vaccine; LGA, Local governmental area; mOPV\#, Monovalent oral polio vaccine type \#; OPV, Oral polio vaccine; RI, Routine immunization; SEIR, Susceptible-exposedinfective-recovered; SIA, Supplemental immunization activity; tOPV, Trivalent oral polio vaccine; WHO, World health organization; WPV\#, Wild polio virus type \# (1/2/3).

\footnotetext{
* Correspondence: kmccarthy@intven.com

'Intellectual Ventures Laboratory, 3150 139th Ave SE, Bellevue, WA 98005,

USA

Full list of author information is available at the end of the article
}

(c) 2016 The Author(s). Open Access This article is distributed under the terms of the Creative Commons Attribution 4.0 International License (http://creativecommons.org/licenses/by/4.0/), which permits unrestricted use, distribution, and reproduction in any medium, provided you give appropriate credit to the original author(s) and the source, provide a link to the Creative Commons license, and indicate if changes were made. The Creative Commons Public Domain Dedication waiver (http://creativecommons.org/publicdomain/zero/1.0/) applies to the data made available in this article, unless otherwise stated. 


\section{Background}

In 1988, the Global Polio Eradication Initiative (GPEI) was launched, and since then, all but three countries (Nigeria, Pakistan, and Afghanistan) have interrupted endemic transmission of all three wild poliovirus (WPV) serotypes [1]. The world has been free of WPV serotype 2 (WPV2) since 1999 [2], and no cases of WPV3 have been reported since 2012 [1]. WPV1 transmission continues uninterrupted in Pakistan and Afghanistan, but the last case in Nigeria was observed on July 24, 2014 [3]. WPV1 paralysis case counts in Nigeria in 2010-2014 were substantially lower than during the 2000s, and this decline in cases has culminated in the announcement, on 25 September, 2015 [4], of the interruption of endemic WPV1 transmission in Nigeria.

This article describes a polio transmission model developed to investigate the interruption of WPV1 transmission in northern Nigeria. This question has been addressed [5-8] using other transmission models; of these, the models presented in $[5,8]$ are not calibrated specifically to the Nigerian context, and indicate that high (>95\%) confidence in WPV1 interruption may require case-free periods of 3 years or more, while $[6,7]$ present models specific to northern Nigeria, and find that as little as 1 year without a WPV1 paralysis case is sufficient to be confident that transmission has been interrupted. The model presented here is structurally distinct from the models of $[6,7]$, and provides further support for their conclusions that interruption can be confidently declared with a shorter case-free period. The model is calibrated to WPV1 case counts in Kano state, Nigeria. Historically, Kano state accounts for approximately $30 \%$ of WPV1 paralytic cases in northern Nigeria (despite having only $\sim 16 \%$ of the total population), and 5 of the 6 cases reported in 2014 were from Kano state. The model is an individual-based model featuring generic susceptible-exposed-infective-recovered (SEIR) dynamics. The model was developed in a datadriven iterative fashion, beginning with a homogenously mixed, single-population SEIR model, to which age structure, seasonal forcing, and spatial transmission are progressively added to improve the ability of the model to reproduce the observed historical data.

\section{Methods}

The generic disease branch of the individual-based disease modeling software EMOD DTK v1.6 was used to model polio transmission. The model was developed in an iterative fashion, beginning with a generic, individualbased SEIR model, with age structure, seasonal forcing, and division into spatial metapopulations progressively added to the model to improve fits to the data. Only children under 5 years of age are included in the model, as these children account for $\sim 98 \%$ of total WPV1 paralytic cases in the dataset for northern Nigeria. The 2006 Nigerian census reports that 1.8 million children under 5 years old lived in Kano state at that time [9]. The simulated birth rate maintains a population growth rate of $2.8 \%$ [10]. Our simulated population consists of approximately $800 \mathrm{k}$ agents in 2006 (averaged over simulation instances, as vital dynamics are stochastic in this model), so that each simulated individual represents approximately 2.2 real individuals. Simulation with 1.8 million agents resulted in unacceptably long instance runtimes. Reducing the simulated population prevented this issue from occurring, and as the model implements frequency-dependent transmission, the mean dynamics should not directly depend on the number of simulated agents, as long as that number is well above the critical community size.

Upon clearance of infection or a successful immunization with oral polio vaccine (OPV), individuals are modeled as being completely immune to future infection. Challenge studies have shown that individuals are in fact susceptible to reinfection. However, the probability of reinfection is strongly reduced after a successful infection or vaccination; the metastudy presented in [11] estimates that the relative odds of shedding serotype 1 poliovirus post-challenge among OPV-vaccinated individuals compared with unvaccinated individuals is 0.13 . [12] presents an odds ratio of 0.15 , with $80 \%$ of unvaccinated individuals excreting virus post-challenge, compared with $37 \%$ of OPV-vaccinated individuals. The OPV-vaccinated individuals in [12] were also observed to shed for a mean of 4.6 days, as opposed to 20.4 days among the unvaccinated, and the mean titer of virus excreted by OPV-vaccinated individuals was over 3 orders of magnitude lower than unvaccinated controls; naturally immune individuals exhibited similar characteristics to the OPV-vaccinated group. The reduced chance of infection, shorter shedding period, and reduced shedding titer of OPV- and WPV-experienced individuals combine to provide support to the simplifying assumption that the observed disease transmission dynamics are driven primarily by naïve individuals, rather than by reinfection of previously individuals.

The historical SIA campaign calendar for northern Nigeria (see Fig. 8) is implemented in simulation. In calibration of this model, the effects of SIA campaigns are parametrized by a campaign coverage multiplied by assumed vaccine take rates for different vaccine types. The product of the two represents the fraction of susceptible children immunized in a single SIA round and is the relevant quantity being calibrated; this product is constrained to lie between 0 and 1 . Model calibration figures will present the campaign coverage alone, as the vaccine take rates are assumed to be constant. Trivalent OPV campaigns are modeled with a $15 \%$ probability of successful vaccination against type 1 , while monovalent 
OPV1 and bivalent OPV are modeled with a $20 \%$ success probability each; these numbers are taken from 30-day seroconversion rates to a birth dose in India [13]. The efficacy of the multiple types of OPV has been measured in numerous studies [11, 13-22], and the results vary considerably across studies. The numbers employed here are on the lower end of observed singledose take rates. Circulation of attenuated vaccine-derived virus is not modeled. Each simulation begins on Jan 1, 1985, with a 5-year period in which infected individuals are stochastically imported into the network. The mean importation rate is one infected individual per week per $10 \mathrm{k}$ population for these 5 years, after which the disease is sufficiently established to survive without importation in the absence of vaccination. The SIA campaign calendar was provided by the Nigerian WHO and [23-26].

\section{Model development and calibration}

The acute flaccid paralysis (AFP) surveillance database, maintained by the Nigerian WHO, provides the basis for model calibration; the model was calibrated only to data from 2003-2009. Only AFP cases with WPV1 shedding confirmed by the Global Polio Laboratory Network [27] are included. Incremental mixture importance sampling (IMIS) [28] was used for parameter space exploration and calibration. Though IMIS applies to deterministic models and true likelihood functions, the authors find it to work quite well at calibrating stochastic models given an objective function, so long as parameter-based variation in the objective function outweighs the stochastic variance in the objective function at a particular parameter set, as is the case for most of the calibrations presented below. The value of the objective functions evaluated at a particular parameter set and stochastic instance will be referred to as the score. The objective functions themselves are described in detail in Additional file 1 . The data targets are the time series of case counts, binned twice per month; the distribution of age at onset of paralysis, binned into 6-month age bins; and the spatial distribution of cases at the LGA level.

The model was developed in an iterative fashion, beginning with a generic, individual-based SEIR model, and adding age structure, seasonal forcing, and division into spatial metapopulations progressively to improve fits and target additional features of the data. The model development and calibration section is split into subsections describing the reasoning and calibration at each step of development, beginning with a very simple (and poor) model and progressing through a couple of intermediate models before arriving at the finalized model. The reader may wish only to see the specification and calibration of the finalized model and results, contained in Additional file 1- Model Specification, Model Development - Spatial Model, and Results, respectively.

\section{Model development - Basic SEIR model}

The incubation and infectious periods, base reproductive number, and the campaign coverage of a basic SEIR model were fit to these data. Campaign coverage is assumed to be constant over this time period, and campaigns are modeled as being randomly accessed by individuals, with no structured heterogeneity in individuals' probabilities of being vaccinated in a single campaign. The infectious and incubation periods are of fixed duration, rather than exponentially distributed. The choice of a fixed duration maintains the benefit of having only a single parameter to calibrate for each, like the exponential distribution, while removing that distribution's probability of individuals drawing unrealistically short infectious durations.

The case-to-infection ratio of WPV1 in immunologically naïve individuals is approximately 1:200 [29]. For the purposes of calibration, the modeled incidence over time is fit to the case count data using a Poisson approximation to a binomial objective function, which is maximized over an unconstrained case-to-infection ratio (for an investigation of overdispersion in the case counts relative to the model, and how this affects the results, please see Additional file 1, Overdispersion section). This construction provides a scale-independent fit of the shape of the modeled incidence curves to the case counts over time. In the high-incidence periods used for calibration and with a large simulated population, the average dynamics are quite insensitive to the total modeled population. More details about the objective functions employed are included in Additional file 1.

Figure 1 presents the results of this calibration. Figure 1(a) and (b) show that the objective score is strongly dependent on $\mathrm{R}_{0}$ and SIA coverage, but relatively weakly dependent on the incubation and infectious periods; the space around 4 days exposed and 5 days infectious is preferred, but individual samples of comparably high score are present throughout the 2D space. Figure 1(c) and (d) present the target data as blue bars. The red contours indicate the $68 \% / 95 \% /$ $99 \%$ quantiles of distributions constructed by weighting the outputs according to their score (were the objective functions true likelihoods, these would represent quantiles of the posterior distribution; as they are not, the statistical interpretation of these contours is not immediately obvious, but they serve to demonstrate how well the calibrated model reproduces the targeted features of the data). These figures demonstrate that the capability of this model to fit the data is rather poor; the age distribution of infection (as expected) is monotonically decreasing, in contrast to the data which peaks in the 1.5-2 year age bin. The simulated case counts over time do exhibit a low-amplitude oscillation with the 2year periodicity exhibited by the data, but fail to 


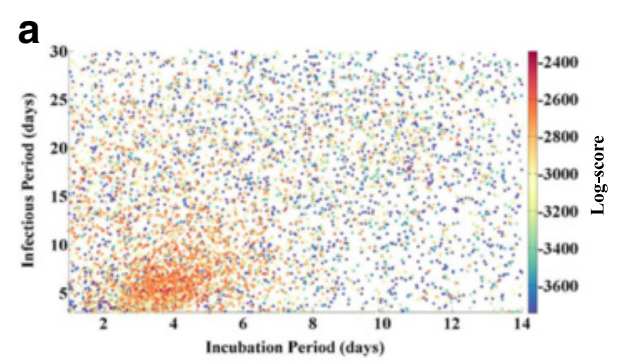

C

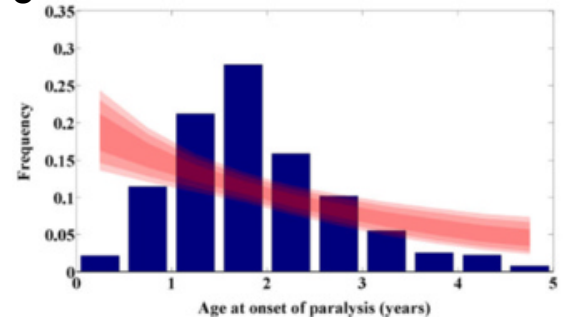

b

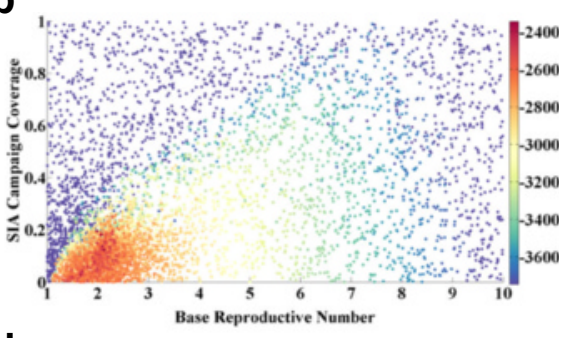

d

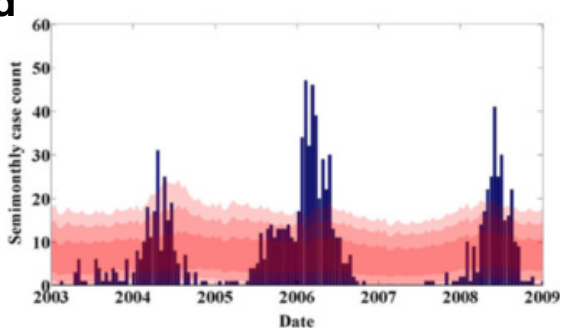

Fig. 1 Calibration of basic SEIR model. a Log-score (color) vs. incubation and infectious period. b Log-score vs. Ro and campaign coverage. c Data (blue) and score-weighted distribution of simulations (68, 95, $99 \%$ quantiles in red) for age distribution of paralysis cases. d Data (blue) and score-weighted distribution of simulations $(68,95,99 \%$ quantiles in red) for time series of case counts

reproduce the high amplitude of the outbreaks and long troughs of very low case counts in the data.

\section{Model development - Age dependent exposure and seasonality}

The poor performance of a basic SEIR model is not unexpected. In a homogenously mixed SEIR model, new infections will be distributed uniformly among the susceptible population, the proportion of susceptibles will decline with age as older age groups have been exposed for longer, and the distribution of new infections will thus decline with age as well, as seen in the simulation results of Fig. 1(a). Similarly, oscillatory behavior in a homogenously-mixed SEIR models is expected to damp toward an equilibrium value; stochastic resonance can drive oscillations at the natural frequency of the system, but for the large population simulated here these oscillations are insufficiently large to explain the data. This model is presented primarily for comparison against more developed models, to demonstrate the value of additional data-driven complexity.

A number of mechanisms could potentially explain the discrepancy in the simulated age at infection distribution and the observed age at onset of paralysis. Age-dependence in the case-to-infection ratio has been reported in the past [30]; however, the measurements indicate no more than a factor of 2 difference over the first 5 years of life, insufficient to transform the simulated distribution into the data. Maternal antibodies are another obvious potential explanation, but previous work indicates that these wane with a half-life on the order of 3-4 weeks [31-35], much too rapidly to solely explain a rising attack rate over the first 2 years of life. The hypothesis implemented into the model is reduced mixing by young children, such that the force of infection is reduced by an age-dependent factor. For simplicity's sake, this age-dependent scaling factor is assumed to be multiplicative, linearly dependent on age, and bounded between 0 and 1 (where 0 represents no exposure to infectivity, and 1 represents full exposure. To clarify, let $\beta(a, t)$ represent the force of infection from all infectives onto susceptibles of age $a$, then:

$$
\beta(a, t)=\left\{\begin{array}{cl}
0, & f_{0}+f_{1} a \leq 0 \\
\beta_{0}\left(f_{0}+f_{1} a\right), & 0<f_{0}+f_{1} a<1 \\
\beta_{0}, & f_{0}+f_{1} a \geq 1
\end{array}\right.
$$

The model is recalibrated, with the slope and age-0 intercept of this scaling factor included as additional dimensions in the calibration.

Figure 2 shows the results of this calibration. The simulated age at infection distribution depends most strongly on the base reproductive number and the slope and intercept of the age-dependent mixing coefficient. Figure 2 only presents the score in the $2 \mathrm{D}$ space of $\mathrm{R}_{0}$ and the slope of the age-dependent mixing; the most likely points for the intercept are clustered near 0 , as expected due to the very low case counts in the 0-6 month age bin. It should be noted that this reduced mixing induces a shift in the true $R_{0}$ that depends on the age-dependent mixing factor and the age distribution of the population; for the remainder of this paper, $R_{0}$ (or base reproductive number in figure axes) will refer to 


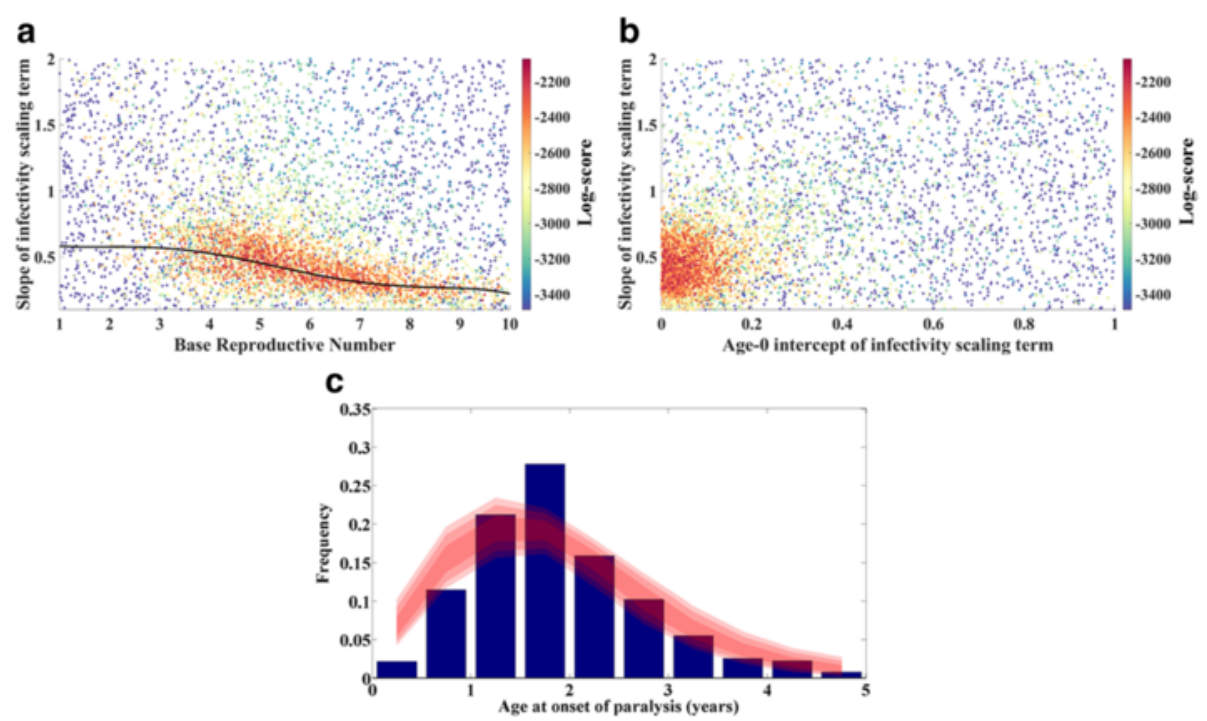

Fig. 2 Calibration of SEIR model w age-dependent transmission. a Log-score (color) vs. base infectivity and the slope of age-dependent susceptibility in early life (described in text). $\mathbf{b}$ Log-score (color) vs. the age-0 intercept and slope of age-dependent susceptibility in early life. c Data (blue) and score-weighted distribution of simulations $(68,95,99 \%$ quantiles in red) for age distribution of paralysis cases

the daily force of infection times the infectious period, which represents the $R_{0}$ within a fully mixed population.

For subsequent calibrations and model runs, the intercept of age-dependent mixing will be fixed at 0 (see Fig. 2 panel b), and the relationship between the slope and the base reproductive number will used to collapse these two onto a single dimension; that is, $R_{0}$ will remain a freely fitted parameter, but at a given $R_{0}$, the age-dependent mixing slope is determined by the fitted curve shown in Fig. 2a.

The next model iteration aims to address the strong periodicity and high amplitude of WPV1 outbreaks, by adding a seasonal forcing term to the infectivity:

$$
\beta \rightarrow \beta\left(1+A\left(\cos \left(\frac{t-t_{0}}{365}\right)\right)\right)
$$

Seasonal outbreaks have been observed in many infectious diseases [36-43], and seasonally-forced models have been shown to exhibit bifurcations from annual outbreaks to dynamics with longer periodicities [44, 45]. The 2-year periodicity of WPV1 outbreaks in Kano state constrains the space of combinations of base reproductive number, SIA campaign coverage, and seasonal forcing amplitude.

Figure 3 shows the results of this calibration procedure. The fit to the case count data is substantially improved by the addition of seasonal forcing to the model, and the fit to the age distribution remains good. The overall peak score has increased dramatically; compare the color bar limits between Fig. 3 and Fig. 1. The structure of the objective function presents some interesting features, with multiple broad regions of high score. The incubation period and infectious period are not strongly constrained by the fits, but existing studies indicate a very short delay from exposure to the onset of viral excretion [46, 47]. When the calibration space is restricted to include only incubation periods between 1 and 5 days, the scoreweighted mean infectious period is 27 days, a value which is also within the range supported by shedding studies $[12,46,48]$. Inclusion of these features appears to have improved the fit of these biological parameters relative to the SEIR model without these additional features, which inferred a mean infectious period of only a few days, Fig. 1. The seasonal forcing model is best fit with an amplitude of 0.11 and a peak on April 10. These values will be fixed in future calibration runs, while $\mathrm{R}_{0}$ and the mean SIA campaign coverage remain as calibration parameters.

As noted early in this section, the time series of cases from data and infections from simulation are compared using a scale-independent fit, with the case-to-infection ratio representing a free scaling parameter in the fit. While this simple SEIR model with minimal added complexity is able to reproduce the outbreak dynamics, the weighted mean case-to-infection ratio is about 1:1650, much lower than the expected 1:200 ratio for WPV1. Assuming the expected 1:200 ratio, the model overpredicts the observed number of cases by a factor of approximately 8 on average. This discrepancy may indicate the existence of some unmodeled complexities. Multiple hypotheses could all plausibly contribute to this discrepancy: The assumption of complete immunity after infection with OPV or WPV rather than the biologically justifiable partial immunity and waning immunity could allow for some degree of transmission to proceed through individuals who are less likely to present 


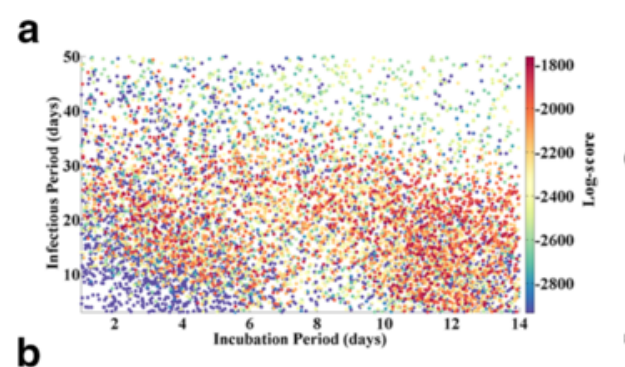

b

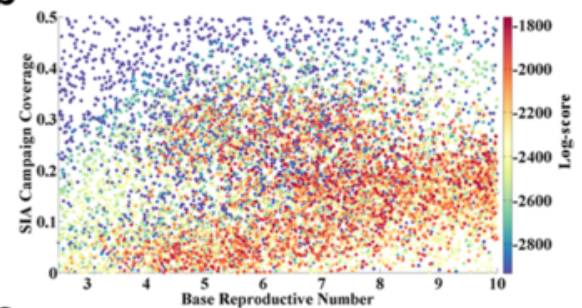

C
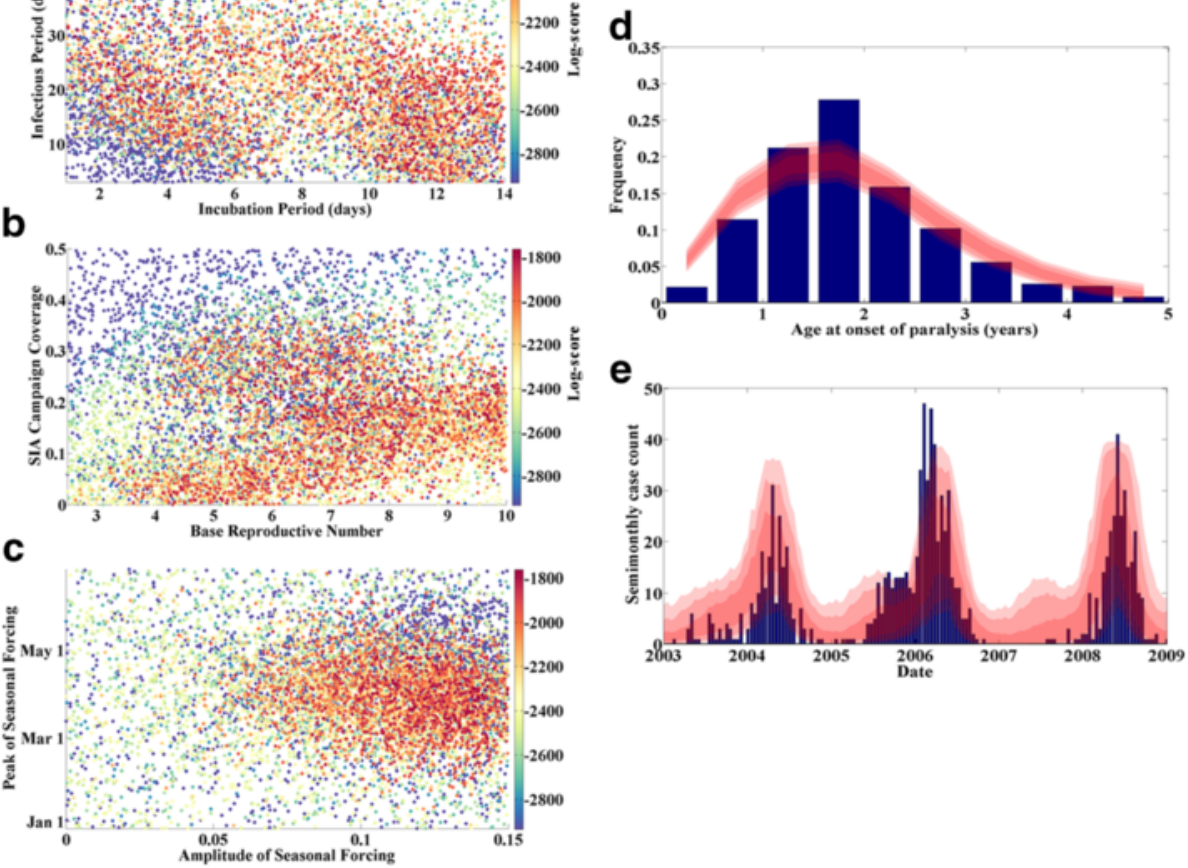

e

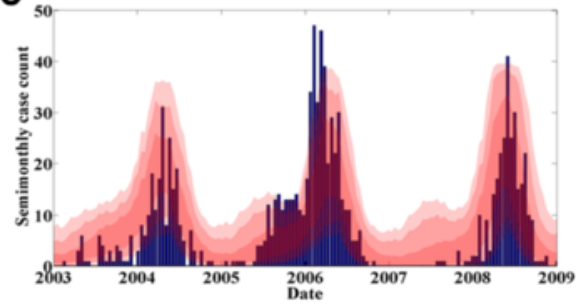

Fig. 3 Calibration of SEIR model w age-dependent transmission and seasonal forcing. a Log-score (color) vs. incubation and infectious periods. b Log-score vs. Ro and campaign coverage. c Log-score (color) vs. the phase and amplitude of seasonal forcing in infectivity. $\mathbf{d}$ Data (blue) and score-weighted distribution of simulations $(68,95,99 \%$ quantiles in red) for age distribution of paralysis cases. e Data (blue) and score-weighted distribution of simulations $(68,95,99 \%$ quantiles in red) for time series of case counts

paralysis (though for reasons given previously, the authors expect this contribution to transmission to be small), individual-level heterogeneity in transmission (through host factors or disparate social networks), and the treatment of Kano as a single mixing population, rather than accounting for the spatial distribution of the population and how this affects transmission. The model development proceeds by investigating this last hypothesis, though all are likely to contribute.

\section{Model development - Spatial model}

The polio case dataset used for calibration reports cases at the scale of local government areas (LGAs), providing spatial information that may also be incorporated into model calibration, which requires splitting the singlepopulation model presented above into a set of metapopulations. Construction of the spatial metapopulation model is covered in detail in Additional file 1. WorldPop $[49,50]$ population maps are the basis for metapopulation construction. Spatially contiguous features of high population density are identified and aggregated into population centers, with the total population placed at the population-weighted mean latitude and longitude. Any remaining low-density background population is aggregated to the nearest population center. Nodes with total populations below 1000 individuals are merged with their nearest neighbor. This process results in 1322 nodes. Each of these population centers is a separate metapopulation, or node, in the model.

Nodes are internally well-mixed (with the agedependent mixing described above), and coupled to each other through migration of individuals. Individuals are assumed to take only round-trip migration, with an average duration of 1 day at the destination node. Individual migration rates are assumed to follow a gravity-like model, with the rate linearly increasing with destination population, inversely linear with the distance to the destination node, and independent of the population of the home node.

$$
R_{i j}=A * \frac{p_{j}}{d_{i j}}
$$

The migration rate is implemented in units of perindividual per day, so that the total flow from node $i$ to node $j$ is implicitly linear in the population of node $i$. The network is not completely connected; migration from a node only occurs to its 8 nearest nodes and its 30 remaining most probable destinations. 
Calibration of the spatial model covers a 5-dimensional parameter space including the coverages of routine immunization (RI) and campaign immunization, the base reproductive number, a coefficient of variation in campaign coverage (though not RI) at the LGA level, and the scaling constant of the overall rate of migration.

The spatial parameters added to the model necessitate the addition of components to the objective function that are sensitive to spatial effects. The new target data include the number of LGAs reporting at least one case within a 3 month time window, and the annual case counts in each of the 44 LGAs of Kano state. The objective functions do not aim to match specific LGAs between simulation and data, but target the general heterogeneity in LGA-level case counts.

Results of the calibration are presented in Fig. 4 (except for the age of infection distribution, suppressed as the fit is of similar quality to those presented previously). Panels a-c present the log-score distributions in 2D planes similar to those shown previously. The best-fit campaign and RI coverages both increase with infectivity, as expected. The LGA-level coefficient of variation of the campaign coverage is not strongly constrained, though the scaling factor on the migration rate is constrained to lie within approximately 1 order of magnitude. To provide an interpretation for this result, Fig. 5 presents a histogram of the unscaled migration rates by node. Scaling this histogram by the high-score region in Fig. 4(c) reveals that the best fits occur when individuals in most nodes have outbound travel frequencies from about once per 3 days to once per 30 days.

The inferred case-to-infection ratio from the metapopulation model was approximately 1:1200. While still much higher than the expected 1:200, this does represent an improvement in the overall scale of the simulated outbreaks when compared with the single-population model. Other unmodeled heterogeneities may be responsible for the continuing discrepancy; perhaps the modeled population may be viewed as an undervaccinated subpopulation or a subpopulation at high risk relative to the total population.

After calibration of the spatial model, the model is applied to the question of elimination. At the time of writing, no cases of WPV1 paralysis have been observed in Nigeria since July 24, 2014. The WHO standard for certifying interruption of WPV in a region is 3 years without an observed paralysis case. Other models have been
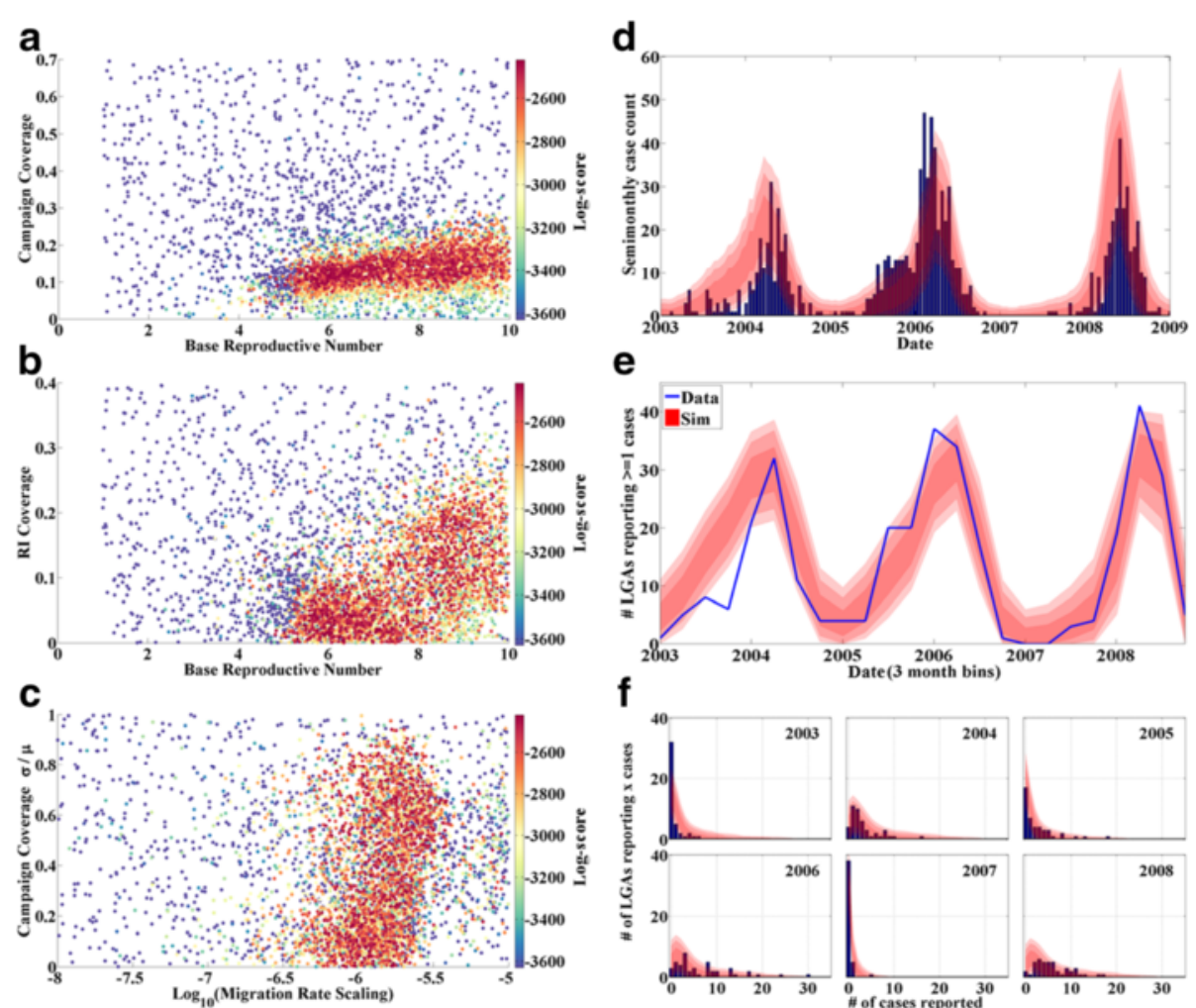

Fig. 4 Calibration of the spatial polio-like model. a Log-score (color) vs. $R_{0}$ and campaign coverage. b Log-score (color) vs. $R_{0}$ and RI coverage. c Log-score (color) vs. scaling term in the migration model (x) and LGA-level coefficient of variation in campaign coverage. $\mathbf{d}$ Data (blue) and score-weighted distribution of simulations (68, 95, $99 \%$ quantiles in red) for time series of case counts. e Data (blue) and score-weighted distribution of simulations $(68,95,99 \%$ quantiles in red) for the number of LGAs reporting at least 1 case in a given time period. $\mathbf{f}$ Data (blue) and score-weighted distribution of simulations (68, 95, $99 \%$ quantiles in red) for distribution of annual case counts among the 44 LGAs 


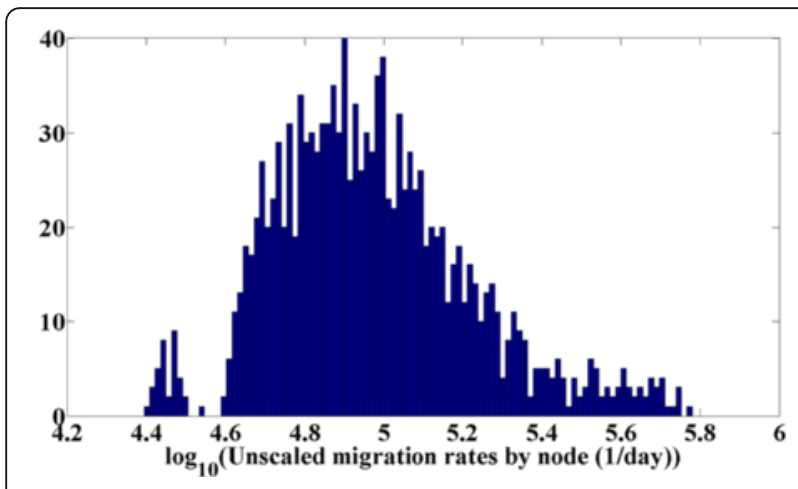

Fig. 5 Histogram of unscaled outbound migration rates by node. The high-score region shown in Fig. 4 centers around a scaling factor of -6 , indicating expected migration rates for most nodes at 0.1 per person per day

applied to the question of elimination probability given a case-free period $[5-8,51]$, and this model is used to address the same question.

The calibration parameter space from the 2003-2009 calibration is resampled for each simulation, with the priors constricted to reflect the regions of high score in Fig. 4. At this stage, the fitting timeframe is extended to include all data until the most recently observed WPV1 case, and the simulation timeframe is extended to 2020. Importation pressure from other states in northern Nigeria is added to the model because Kano state underwent a period of 20 months in 2009-2010 without a single WPV1-positive paralysis case. This is a lowprobability event in this model and indicates the possibility of a local elimination followed by reimportation.

Increasing campaign coverage over time is also added to the model for this investigation, as the available data on campaign coverage in Kano indicates that campaign coverage improved in the late 2000s and into the 2010s (lot quality assurance sampling (LQAS) data, provided by the Nigerian WHO). In this exploration, campaign coverage is assumed to increase linearly with time, beginning at some time between Jan 1, 2005 and Jan 1, 2010. After the last campaign in the historical and planned campaign calendar (Dec. 2015), campaigns are assumed to use bOPV or mOPV1, with campaigns occurring at a constant rate of $9 /$ year.

Finally, the scale-free fitting utilized in calibration is not appropriate for addressing the question of pathogen elimination, as the size of the mixing population and the caseto-infection ratio affect how long infection can silently transmit. Therefore, the simulated population is reduced to match the scale of the simulated infections with the observed paralysis data at a case-to-infection ratio of 1:200. The final simulated population is 284000 in Mar 2006, compared to the census population of $0-5$ year olds of 1.8 million in the same year. Figure 7 demonstrates that the quality of the calibration fits remains comparable to the larger population simulations.

\section{Results and discussion}

The probability that WPV1 has been eradicated at day $t$ (i.e., the number of infected $I(t)=0$ at day $t$ ), given that the cumulative number of cases observed $N_{c}(t)$ since the last case is 0 , is:

$$
\begin{aligned}
P_{\text {erad }}(t) & =P\left(I(t)=0 \mid N_{c}(t)=0\right) \\
& =\frac{P\left(N_{c}(t)=0 \mid I(t)=0\right) P(I(t)=0)}{P\left(N_{c}(t)=0\right)}
\end{aligned}
$$

The probability of observing 0 cumulative cases is a binomial draw from the cumulative number of infections $I_{c}(t)$, and $P(I(t)=0)$ is an indicator function that is either 0 or 1 for each simulation and time point.

$$
\begin{aligned}
P_{\text {erad }}(t) & =\frac{P\left(N_{c}(t)=0 \mid I(t)=0\right)}{P\left(N_{c}(t)=0\right)} \\
& =\frac{\sum_{\{\operatorname{sims} \mid I(t)=0\}} \operatorname{Bin}\left(0 \mid I_{c}(t), .005\right)}{\sum_{\{\text {all sims }\}} \operatorname{Bin}\left(0 \mid I_{c}(t), .005\right)}
\end{aligned}
$$

A total of 16,350 simulations were performed, of which 8113 survive until the time of the most recently observed case. Figure 6 presents the probability of elimination vs. time since the last case. One year out from the last case, this model predicts a $91 \%$ probability of elimination, which rises to nearly $99 \% 2$ years out.

Figure 7 presents a selection of slices in the score space and comparisons of simulations to data; Fig. 7(a) presents the log-score in the space of the migration rate scaling and the coefficient of variation of campaign coverage. A comparison with Fig. 4 reveals that including the years from 2009-2014 and the rise in campaign coverage over time allows for stronger constraints on this heterogeneity; the most likely values are quite high, which allows local eliminations in the most well covered

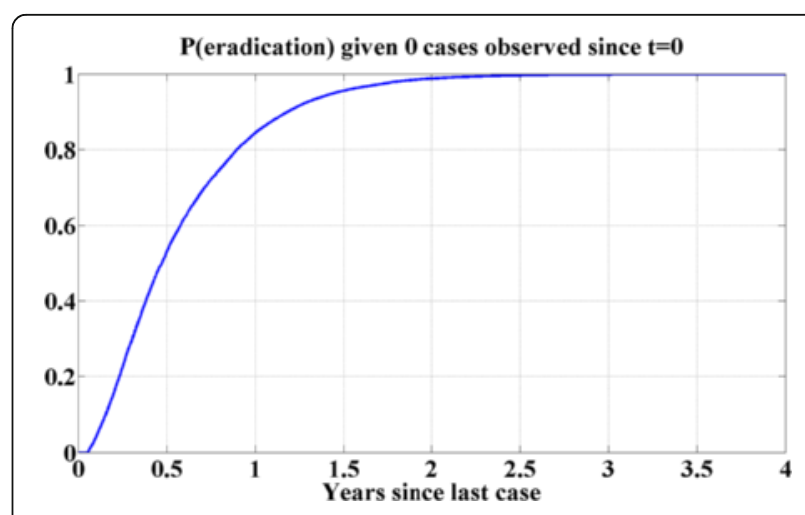

Fig. 6 Estimated probability of WPV1 elimination given 0 cases observed since the last confirmed WPV1 case (Jul 24, 2014 in Kano state) 
a

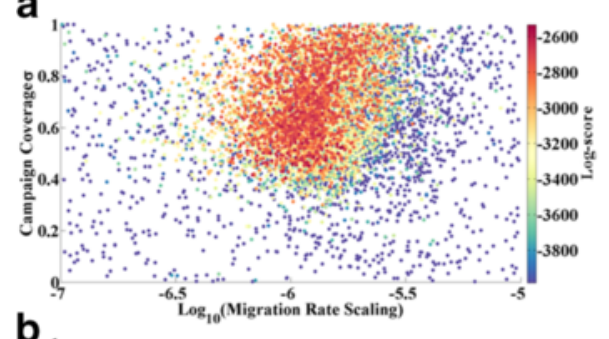

b

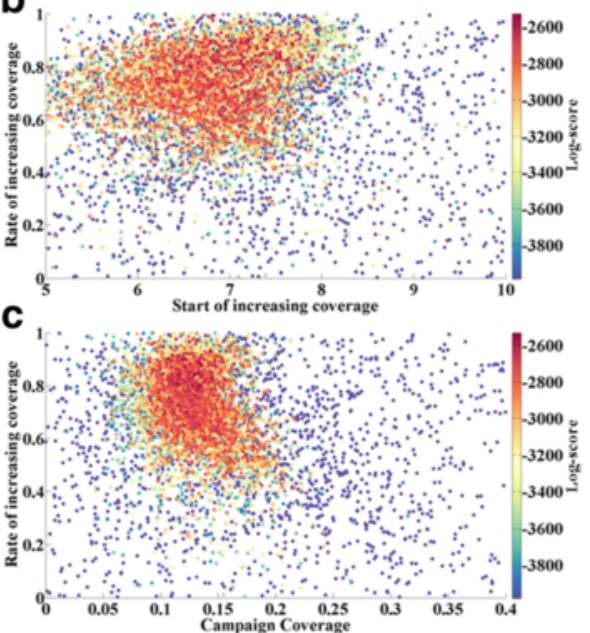

d
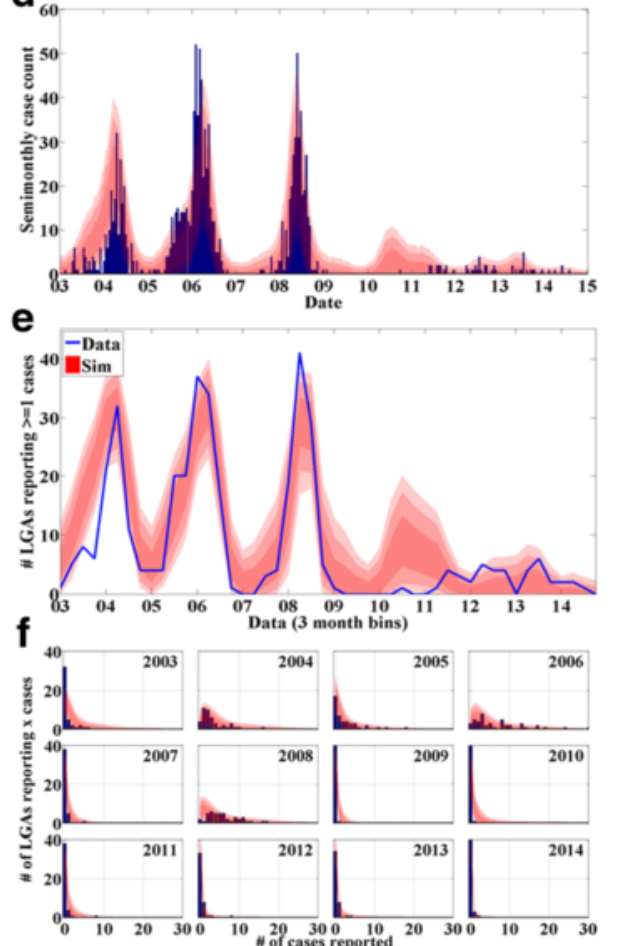

Fig. 7 Results of date of elimination simulations. a Log-score vs. the scaling factor in the migration rate model and the LGA-level coefficient of variation in campaign coverage. $\mathbf{b}$ Log-score vs. the time at which campaign coverage begins to increase, and the relative annual rate of increase. c Log-score vs. the base campaign and the relative annual rate of coverage increase. $\mathbf{d}$ Data (blue) and score-weighted distribution of simulations $(68,95,99 \%$ quantiles in red) for age distribution of paralysis cases. e Data (blue) and score-weighted distribution of simulations (68, $95,99 \%$ quantiles in red) for time series of case counts. $\mathbf{f}$ Data (blue) and score-weighted distribution of simulations (68, $95,99 \%$ quantiles in red) for the number of LGAs reporting at least 1 case in a given time period

LGAs but continued circulation in the set of poorly covered LGAs. The model fits best when campaign coverage begins to increase sometime between 2006 and 2008, with quite rapid increases of $50-100 \%$ per year. The increase in coverage, as implemented, is relative to the "base" coverage, which ranges from 10-15\%. The bestfit absolute rates of coverage increase can be estimated from Fig. 7(c) and range from about 5 to $15 \%$ additional absolute coverage (vaccine efficacy remains constant) per year.

Figure 8 provides a more detailed illustration of the fraction of susceptibles who are successfully immunized in each SIA round over time. The model predicts quite low values of immunization within this population throughout the early-mid 2000s. These rates are sufficient to drive the system into sharp peaks of 2-year periodicity, but the calibrated immunization rate rises rapidly through the late 2000 s to match the much lower case counts of 2010-2015. A portion of the posterior distribution of the statewide rate exceeds $20 \%$ beginning in 2014, indicating that the chosen values for take rate may have been too low (as campaign coverage should not exceed $100 \%$ ).
A number of features of this model may contribute to an overall optimistic picture of elimination, however. The campaign coverage is assumed to have been rising over time for approximately the past 10 years, which allows the model to fit the dramatically reduced incidence from 2010-2015 compared to the previous decade, and this increasing coverage is assumed to continue into the future. Furthermore, future modeled campaigns were assumed to be mOPV1 or bOPV, but as the WPV1 case-free period grows, the use of tOPV, which is less effective at providing immunity against Type 1 [13], has increased correspondingly as the focus shifts to eliminating circulating vaccinederived poliovirus Type 2 (cVDPV2). The closed nature of the modeled population also does not admit the possibility of exportation to at-risk populations connected to that of Kano state; even if transmission is currently declining towards elimination, exportation to outside populations could provide a reservoir for re-establishment. Time-varying or LGA-specific levels of routine immunization are not accounted for in this model; nor is the co-administration of inactivated polio vaccine (IPV) with OPV.

The probability of polio elimination given a case-free period has been addressed in previous work, in other 


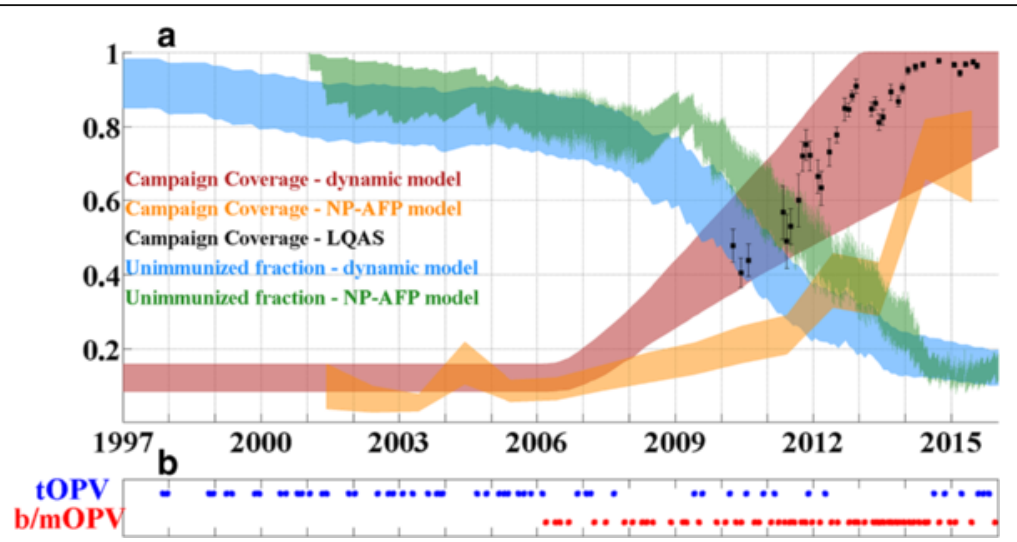

Fig. 8 a $95 \%$ quantiles of the score-weighted distributions of (red) statewide mean SIA coverage over time as estimated in calibration of the presented model; (orange) statewide mean SIA coverage over time as estimated using a model based on non-polio acute flaccid paralysis (NP-AFP); (black) statewide mean SIA coverage as estimated from lot quality assurance sampling (data provided by Nigerian WHO); (blue) statewide mean vaccine-derived immunity in 0-5 year olds from the presented model; (green) statewide mean vaccine-derived immunity in $0-5$ year olds from a statistical model deriving campaign coverage from the reported vaccine dose histories of non-polio acute flaccid paralysis cases. The NP-AFP immunity model is an extension of that presented in [56] (publication detailing newer model is in preparation). $\mathbf{b}$ Illustration of historical campaign calendar in Kano state

contexts $[8,51]$ and in Nigeria [5-7]. The results of this model are similar to other recently published results considering the Nigerian context, which are derived from models differing widely in structure from the present model. Of these models, the present model uniquely features spatially structured metapopulations with heterogeneous immunization coverage, which admits certain phenomena of interest in the near-elimination regime - localization of transmission chains and the possibility of escape from an infected region to another region that is not experiencing local transmission. The calibration procedure presented is also unique among the Nigeria models in methodology and in the data targets. The confluence of results from multiple models provides some confidence that the optimistic biases discussed above do not drive this result, and provide support to the WHO's recent removal of Nigeria from the list of polio-endemic countries [4].

\section{Conclusions}

The model predicts that at the time of writing, October 2015, the lack of any cases since July 24, 2014 [3] indicates a probability of $91 \%$ that WPV1 has been eliminated from Kano state. The other states in Nigeria have experienced even longer case-free periods (the only other state with a WPV1 case in 2014 was Yobe, on April 19, 2014), and as Nigeria is the last remaining country in Africa to experience endemic transmission of WPV1, this probability can be interpreted as an upper bound on the probability that endemic transmission of WPV1 has been interrupted continent-wide. If another year passes without any observed WPV1 paralysis cases, this model indicates a $99 \%$ chance of elimination.
The model assumes that frequent SIAs with high coverage continue to take place in Kano state through the end of the simulation period, and though these results are optimistic that WPV1 transmission in Kano has been or will soon be interrupted, these results do not provide support for any reduction in vaccination activities in the immediate future. The results indicate that it is important for the polio program to maintain vigilance in surveillance and vaccination activities to prevent WPV1 resurgence through the WHO's 3 -year eradication certification period.

Added June 2016: On 23 March 2016, a cVDPV2positive environmental sample was isolated in the Maiduguri district of Borno state, Nigeria, and determined to be genetically linked to a strain last observed in May 2014 [52]. As calibration of the presented model focused exclusively on WPV1, it cannot be directly applied to silent circulation of cVDPV2; investigations of silent cVDPV2 circulation can be found in $[6,7]$. Both references find that long-term silent circulation of Type 2 is more likely than Type 1, even with perfect AFP surveillance. The difference in case-to-infection ratios (approximately 1:200 for Type 1, 1:1900 for Type 2 [29]) likely accounts for much of the difference in silent circulation of the two serotypes, but the appearance of this environmental sample demonstrates that silent circulation remains a major concern in certifying global poliovirus eradication, particularly after the April 2016 worldwide withdrawal of OPV2 [53].

Added August 2016: Since preparation of this manuscript, multiple persons infected with WPV1 were discovered in areas of Borno state previously inaccessible to programmatic activities due to violence and instability. One WPV1-positive paralysis case was found along with 
multiple WPV1-positive healthy close contacts, and a second paralysis case that was WPV1-negative was found to have a WPV1-positive close contact [54]. Genetic sequencing indicates a period of approximately 5 years between the virus causing these infections and the most recent known related infection [55]. These observations of WPV1 follow a recent discovery of a cVDPV2 circulating silently for nearly 2 years. These discoveries emphasize the necessity of high-quality surveillance during the polio endgame and certification process. Many other regions of Borno remain inaccessible to surveillance, limiting knowledge about the extent of the WPV1 transmission within the state.

This approximately 5 years of silent circulation may call into question the presented results. The model's predicted probability of elimination vs. time since the last paralytic case depends on two crucial assumptions: that SIAs continue to take place after the last observed paralysis case, and that surveillance for new paralytic cases is of high quality. Neither of these assumptions hold in the inaccessible areas of Borno. This and other models approaching the question all find 5 years of circulation without generating a new paralysis case extremely unlikely [5-8], indicating a probable surveillance failure throughout the inaccessible regions of Borno. These cases, along with the cVDPV2 previously observed in Borno, highlight the existential risk that inaccessible populations present to polio eradication.

\section{Additional file}

Additional file 1: Includes descriptions of model specification, definitions of the objective functions in calibration, investigations of overdispersion, and construction of the metapopulation model. (DOCX $2691 \mathrm{~kb}$ )

\section{Acknowledgements}

We are very grateful for the support of Dr. Andrew Etsano of the Nigeria National Primary Health Care Development Agency, without whom the article would not be possible nor as relevant. We also thank Michael Galway of the Bill and Melinda Gates Foundation for his support of our work in Nigeria and critical feedback on how to make our analyses more useful. We thank Philip Eckhoff, Hil Lyons, Alex Upfill-Brown, Daniel Klein and other colleagues of the Institute for Disease Modeling for helpful discussion on methods and results. The authors would like to thank Bill and Melinda Gates for their active support of this work and their sponsorship through the Global Good Fund. Finally, we would like to thank all those in Nigeria involved in AFP surveillance and laboratory testing.

\section{Funding}

KM and GC are supported by Bill and Melinda Gates through the Global Good Fund. The funders had no role in study design, data collection and analysis, decision to publish, or preparation of the manuscript.

\section{Availability of data and material}

The simulation executable, example input files, and summary of simulation outputs are available via GitHub at https:/github.com/KevinMcCarthyAtIDM/ NigeriaEradication_2016Paper_Repo. The current source code of the EMOD Disease Transmission Kernel and supporting documentation are available at http://idmod.org/idmdoc/\#EMOD/EMODBuildAndRegression/Building EMODTOC.htm. The data utilized in this study was obtained under an exclusive data sharing agreement and is not currently publicly available.

\section{Authors' contributions}

KM designed and ran all simulations and drafted the manuscript. GCC helped conceive of the study and contributed to the manuscript. FS assisted in data access and interpretation of the results and contributed to the manuscript. All authors read and approved the final manuscript.

\section{Competing interests}

The authors declare that they have no competing interests.

\section{Consent for publication}

Not Applicable.

\section{Ethics approval and consent to participate}

Permission for access to the Polio Information System (POLIS) case and campaign databases was granted to the Institute for Disease Modeling researchers through the World Health Organization.

\section{Author details}

${ }^{1}$ Intellectual Ventures Laboratory, 3150 139th Ave SE, Bellevue, WA 98005, USA. ${ }^{2}$ Federal Ministry of Health, Abuja, Nigeria.

Received: 20 November 2015 Accepted: 6 September 2016

Published online: 29 September 2016

\section{References}

1. Kew OM, Cochi SL, Jafari H, Wassilak SGF, Mast EE, Ousmane MD, et al. Possible Eradication of Wild Poliovirus Type 3 - Worldwide, 2012. Morb Mortal Wkly Rep [Internet]. 2014 [cited 2015 Sep 16];63:1031-3. Available from: http://www.cdc.gov/mmwr/preview/mmwrhtml/mm6345a4.htm.

2. Apparent Global Interruption of Wild Poliovirus Type 2 Transmission. Morb Mortal Wkly Rep [Internet]. 2001 [cited 2015 Sep 16];50:222-4. Available from: http://www.cdc.gov/mmwr/preview/mmwrhtml/mm5012a2.htm.

3. Global Polio Eradication Initiative: Polio this week; 2 Sept 2015 [Internet] 2015 [cited 2015 Sep 7]. Available from: https://web.archive.org/web/ 20150907180505/http://www.polioeradication.org/Dataandmonitoring/ Poliothisweek.aspx.

4. World Health Organization. World Health Organization. WHO Removes Nigeria from Polio-Endemic List. 2015. [Internet]. World Health Organization; 2015 [cited 2015 Oct 17]. Available from: https://web.archive.org/web/ 20151017231408/http://www.who.int/mediacentre/news/releases/2015/ nigeria-polio/en/.

5. Kalkowska DA, Duintjer Tebbens RJ, Thompson KM. The probability of undetected wild poliovirus circulation after apparent global interruption of transmission. Am J Epidemiol [Internet]. 2012 [cited 2015 Aug 14];175:93649. Available from: http://www.ncbi.nlm.nih.gov/pubmed/22459121.

6. Kalkowska DA, Duintjer Tebbens RJ, Pallansch MA, Cochi SL, Wassilak SGF, Thompson KM. Modeling undetected live poliovirus circulation after apparent interruption of transmission: implications for surveillance and vaccination. BMC Infect Dis [Internet]. 2015 [cited 2015 Apr 14];15:66. Available from: http://www.biomedcentral.com/1471-2334/15/66.

7. Famulare M. Has wild poliovirus been eliminated from Nigeria? arXiv preprint [Internet]. 2015 [cited 2015 Sep 15]; Available from: http://arxiv.org/ abs/1504.02751

8. Eichner M, Dietz K. Eradication of poliomyelitis: when can one be sure that polio virus transmission has been terminated? Am J Epidemiol [Internet]. 1996 [cited 2015 Aug 14];143:816-22. Available from: http://www.ncbi.nlm. nih.gov/pubmed/8610692

9. Nigerian National Population Commission. 2006 Population and Housing Census, Priority Table Volume IV: Population Distribution by Age and Sex (State and Local Government Area). 2010;1-371. Available from: http://www. population.gov.ng/images/PrioritytableVol4.pdf. Accessed 5 Aug 2015.

10. The World Bank. Population growth (annual \%) [Internet]. World Dev Indic. 2014 [cited 2015 Jan 1]. Available from: http://data.worldbank.org/indicator/ SP.POP.GROW.

11. Hird TR, Grassly NC. Systematic review of mucosal immunity induced by oral and inactivated poliovirus vaccines against virus shedding following oral poliovirus challenge. PLoS Pathog [Internet]. Public Library of Science; 2012 [cited 2015 Jul 7];8:e1002599. Available from: http://journals.plos.org/ plospathogens/article?id=10.1371/journal.ppat.1002599.

12. Ghendon Y, Sanakoyeva I. Comparison of the resistance of the intestina tract to poliomyelitis virus (Sabin's strains) in persons after naturally and 
experimentally acquired immunity. Acta Virol [Internet]. 1961 [cited 2015 Sep 17]; Available from: https://scholar.google.com/scholar?q=+Acta+Virol $+1961+\% 3 B 5 \% 3 A 265-73 \& b t n G=\& h l=e n \& a s \_s d t=0 \% 2 C 48 \# 0$.

13. Sutter RW, John TJ, Jain H, Agarkhedkar S, Ramanan PV, Verma H, et al. Immunogenicity of bivalent types 1 and 3 oral poliovirus vaccine: a randomised, double-blind, controlled trial. Lancet (London, England) [Internet]. 2010 [cited 2015 Jul 7];376:1682-8. Available from: http://www. ncbi.nlm.nih.gov/pubmed/20980048.

14. Grassly NC, Fraser C, Wenger J, Deshpande JM, Sutter RW, Heymann DL, et al. New strategies for the elimination of polio from India. Science [Internet]. 2006 [cited 2015 Sep 16];314:1150-3. Available from: http://www. sciencemag.org/content/314/5802/1150.abstract.

15. John TJ. Antibody response of infants in tropics to five doses of oral polio vaccine. Br Med J [Internet]. 1976 [cited 2015 Sep 16];1:812. Available from: http://www.pubmedcentral.nih.gov/articlerender.fcgi?artid=1639412\&tool= pmcentrez\&rendertype=abstract.

16. John TJ, Jayabal P. Oral polio vaccination of children in the tropics. I. The poor seroconversion rates and the absence of viral interference. Am J Epidemiol [Internet]. 1972 [cited 2015 Sep 16];96:263-9. Available from: http://www.ncbi.nlm.nih.gov/pubmed/4342327.

17. Heymann DL, Murphy K, Brigaud M, Aymard M, Tembon A, Maben GK. Oral poliovirus vaccine in tropical Africa: greater impact on incidence of paralytic disease than expected from coverage surveys and seroconversion rates. Bull World Health Organ [Internet]. 1987 [cited 2015 Sep 13];65:495-501. Available from: http://www.pubmedcentral.nih.gov/articlerender.fcgi?artid= 2491033\&tool=pmcentrez\&rendertype=abstract.

18. Patriarca PA, Wright PF, John TJ. Factors Affecting the Immunogenicity of Oral Poliovirus Vaccine in Developing Countries: Review. Clin Infect Dis [Internet]. 1991 [cited 2015 Aug 25];13:926-39. Available from: http://cid. oxfordjournals.org/content/13/5/926.abstract.

19. Jenkins HE, Aylward RB, Gasasira A, Donnelly CA, Mwanza M, Corander J, et al. Implications of a circulating vaccine-derived poliovirus in Nigeria. N Engl J Med [Internet]. 2010 [cited 2015 Oct 29];362:2360-9. Available from: http:// www.ncbi.n/m.nih.gov/pubmed/20573924.

20. Jenkins HE, Aylward RB, Gasasira A, Donnelly CA, Abanida EA, KoleoshoAdelekan T, et al. Effectiveness of immunization against paralytic poliomyelitis in Nigeria. N Engl J Med [Internet]. 2008 [cited 2015 Oct 29];359:1666-74. Available from: http://www.ncbi.n/m.nih.gov/pubmed/18923171.

21. Mangal TD, Aylward RB, Mwanza M, Gasasira A, Abanida E, Pate MA, et al. Key issues in the persistence of poliomyelitis in Nigeria: a case-control study. Lancet Glob Heal [Internet]. 2014 [cited 2015 Oct 9];2:e90-7. Available from: http://www.ncbi.nlm.nih.gov/pubmed/25104665.

22. El-Sayed N, El-Gamal Y, Abbassy A-A, Seoud I, Salama M, Kandeel A, et al. Monovalent type 1 oral poliovirus vaccine in newborns. N. Engl. J. Med. Mass Medical Soc. 2008;359:1655-65.

23. Progress Toward Poliomyelitis Eradication - Nigeria, 1996-1998. Morb Mortal Wkly Rep [Internet]. 1999 [cited 2015 Sep 16];48:312-6. Available from: http://www.cdc.gov/mmwr/preview/mmwrhtml/00056970.htm.

24. Progress Toward Poliomyelitis Eradication - African Region, 1998-April 1999. Morb Mortal Wkly Rep [Internet]. 1999 [cited 2015 Sep 16];48: 513-8. Available from: http://www.cdc.gov/mmwr/preview/mmwrhtml/ mm4824a2.htm.

25. Progress Toward Global Poliomyelitis Eradication - 1997-1998. Morb Mortal Wkly Rep [Internet]. 1999 [cited 2015 Sep 16];48:416-21. Available from: http://www.cdc.gov/mmwr/preview/mmwrhtml/mm4820a2.htm.

26. Progress Toward Poliomyelitis Eradication - Africa, 1996. Morb Mortal Wkly Rep [Internet]. 1997 [cited 2015 Sep 16];46:321-5. Available from: http:// www.cdc.gov/mmwr/preview/mmwrhtml/00047240.htm.

27. Hull BP, Dowdle WR. Poliovirus surveillance: building the global Polio Laboratory Network. J Infect Dis [Internet]. 1997 [cited 2015 Sep 16];175 Suppl:S113-6. Available from: http://www.ncbi.nlm.nih.gov/pubmed/ 9203702.

28. Raftery AE, Bao L. Estimating and Projecting Trends in HIV/AIDS Generalized Epidemics Using Incremental Mixture Importance Sampling. Biometrics [Internet]. 2010 [cited 2015 Sep 16];66:1162-73. Available from: http://www.pubmedcentral.nih.gov/articlerender.fcgi?artid= 2925068\&tool=pmcentrez\&rendertype=abstract.

29. Nathanson N, Kew OM. From emergence to eradication: the epidemiology of poliomyelitis deconstructed. Am J Epidemiol [Internet]. 2010 [cited 2015 Jun 6];172:1213-29. Available from: http://www.pubmedcentral.nih.gov/ articlerender.fcgi?artid=2991634\&tool=pmcentrez\&rendertype=abstract.
30. Anderson RM, May RM. Infectious Diseases of Humans: Dynamics and Control [Internet]. Oxford University Press; 1992 [cited 2015 Sep 16]. Available from: https://global.oup.com/academic/product/infectiousdiseases-of-humans-9780198540403?cc=us\&lang=en\&.

31. Halsey N, Galazka A. The efficacy of DPT and oral poliomyelitis immunization schedules initiated from birth to 12 weeks of age. Bull World Health Organ [Internet]. 1985 [cited 2015 Sep 16];63:1151-69. Available from: http://www. pubmedcentral.nih.gov/articlerender.fcgi?artid=2536468\&tool= pmcentrez\&rendertype=abstract.

32. Behrend MR, Hu H, Nigmatulina KR, Eckhoff P. A quantitative survey of the literature on poliovirus infection and immunity. Int J Infect Dis [Internet]. 2014 [cited 2015 Jul 31];18:4-13. Available from: http://www.sciencedirect. com/science/article/pii/S120197121300297X.

33. Plotkin SA, Koprowski H, Stokes J. Clinical trials in infants of orally administered attenuated poliomyelitis viruses. Pediatrics [Internet]. 1959 [cited 2015 Sep 16];23:1041-62. Available from: http://pediatrics. aappublications.org/content/23/6/1041.abstract.

34. Gelfand HM, Fox JP, LeBlanc DR, Elveback L. Studies on the development of natural immunity to poliomyelitis in Louisiana. V. Passive transfer of polioantibody from mother to fetus, and natural decline and disappearance of antibody in the infant. J Immunol [Internet]. 1960 [cited 2015 Sep 17];85: 46-55. Available from: http://www.ncbi.nlm.nih.gov/pubmed/13827179.

35. Dong DX, Hu XM, Liu WJ, Li JS, Jin YC, Tan SG, et al. Immunization of neonates with trivalent oral poliomyelitis vaccine (Sabin). Bull. World Health Organ [Internet]. 1986 [cited 2015 Sep 17];64:853-60. Available from: http:// www.pubmedcentral.nih.gov/articlerender.fcgi?artid=2490980\&tool= pmcentrez\&rendertype=abstract.

36. Stone L, Olinky R, Huppert A. Seasonal dynamics of recurrent epidemics. Nature. 2007:446:533-6.

37. Bharti N, Tatem AJ, Ferrari MJ, Grais RF, Djibo A, Grenfell BT. Explaining seasonal fluctuations of measles in Niger using nighttime lights imagery. Science [Internet]. 2011 [cited 2015 Jun 8];334:1424-7. Available from: http://www.pubmedcentral.nih.gov/articlerender.fcgi?artid=3891598\&tool= pmcentrez\&rendertype=abstract.

38. Ferrari MJ, Djibo A, Grais RF, Bharti N, Grenfell BT, Bjornstad ON. Rural-urban gradient in seasonal forcing of measles transmission in Niger. Proc Biol Sci [Internet]. 2010 [cited 2015 Jun 8];277:2775-82. Available from: http://rspb. royalsocietypublishing.org/content/277/1695/2775.short.

39. Grassly NC, Fraser C. Seasonal infectious disease epidemiology. Proc Biol Sci [Internet]. 2006 [cited 2015 Aug 12];273:2541-50. Available from: http:// www.pubmedcentral.nih.gov/articlerender.fcgi?artid=1634916\&tool= pmcentrez\&rendertype=abstract.

40. Grenfell BT, Bolker BM. Kleczkowski a. Seasonality and Extinction in Chaotic Metapopulations. Proc R Soc B Biol Sci. 1995;259:97-103.

41. Dowell SF, Whitney CG, Wright C, Rose CE, Schuchat A. Seasonal patterns of invasive pneumococcal disease. Emerg Infect Dis [Internet]. 2003 [cited 2015 Sep 17];9:573-9. Available from: http://www.pubmedcentral.nih.gov/ articlerender.fcgi?artid=2972762\&tool=pmcentrez\&rendertype=abstract.

42. Dushoff J, Plotkin JB, Levin SA, Earn DJD. Dynamical resonance can account for seasonality of influenza epidemics. Proc Natl Acad Sci USA [Internet]. 2004 [cited 2015 Jul 29];101:16915-6. Available from: http://www.pnas.org/ content/101/48/16915.abstract.

43. Sultan B, Labadi K, Guégan J-F, Janicot S. Climate drives the meningitis epidemics onset in west Africa. PLoS Med [Internet]. Public Library of Science; 2005 [cited 2015 Sep 17];2:e6. Available from: http://journals.plos. org/plosmedicine/article?id=10.1371/journal.pmed.0020006.

44. Keeling MJ, Rohani P, Grenfell BT. Seasonally forced disease dynamics explored as switching between attractors. Phys D Nonlinear Phenom [Internet]. 2001 [cited 2015 Sep 17];148:317-35. Available from: http://www. sciencedirect.com/science/article/pii/S0167278900001871.

45. Altizer S, Dobson A, Hosseini P, Hudson P, Pascual M, Rohani P. Seasonality and the dynamics of infectious diseases. Ecol Lett [Internet]. 2006 [cited 2015 May 18];9:467-84. Available from: http://www.ncbi.nlm.nih.gov/ pubmed/16623732.

46. Alexander JP, Gary HE, Pallansch MA. Duration of poliovirus excretion and its implications for acute flaccid paralysis surveillance: a review of the literature. J Infect Dis [Internet]. 1997 [cited 2015 Aug 31];175 Suppl :S17682. Available from: http://www.ncbi.nlm.nih.gov/pubmed/9203713.

47. Dick GW, Dane DS, McAlister J, Briggs M, Nelson R, Field CM. Vaccination against poliomyelitis with live virus vaccines. 7. Effect of previous Salk vaccination on virus excretion. Br Med J [Internet]. 1961 [cited 2015 Sep 17]; 
2:266-9. Available from: http://www.pubmedcentral.nih.gov/articlerender. fcgi?artid=1969644\&tool=pmcentrez\&rendertype=abstract.

48. Plotkin S, Orenstein WA, Offit P. Vaccines [Internet]. 6th ed. Elsevier; 2013 [cited 2015 Sep 17]. Available from: http://store.elsevier.com/Naccines/ Stanley-Plotkin/isbn-9781455700905/.

49. Linard C, Gilbert M, Snow RW, Noor AM, Tatem AJ. Population distribution, settlement patterns and accessibility across Africa in 2010. PLoS One [Internet]. Public Library of Science; 2012 [cited 2015 Jun 18];7:e31743. Available from: http://journals.plos.org/plosone/article?id=10.1371/journal. pone.0031743.

50. Worldpop - Selected Data: Africa $>$ Nigeria $>$ Population [Internet]. [cited 2015 Sep 17]. Available from: http://www.worldpop.org.uk/data/summary/ ?contselect=Africa\&countselect=Nigeria\&typeselect=Population.

51. Debanne SM, Rowland DY. Statistical certification of eradication of poliomyelitis in the Americas. Math Biosci [Internet]. 1998 [cited 2015 Aug 14];150:83-103. Available from: http://www.sciencedirect.com/science/ article/pii/S0025556498000078.

52. Global Polio Eradication Initiative: Key Countries, Nigeria; June 20, 2016 [Internet]. Available from: https://web.archive.org/web/20160620214959/ http://www.polioeradication.org/Keycountries/Nigeria\%28cVDPV\%29.aspx. Accessed 18 Aug 2016.

53. Patel M, Orenstein W. A World Free of Polio - The Final Steps. N Engl J Med [Internet]. 2016 [cited 2016 Jun 21];374:501-3. Available from: http:// www.nejm.org/doi/abs/10.1056/NEJMp1514467.

54. Global Polio Eradication Initiative. Polio this week as of 17 August 2016 [Internet]. 2016 [cited 2016 Aug 18]. Available from: https://web.archive.org/ web/20160818185304/http://www.polioeradication.org/dataandmonitoring/ poliothisweek.aspx

55. World Health Organization Media Centre. Government of Nigeria reports 2 wild polio cases, first since July 2014 [Internet]. WHO. World Health Organization; 2016 [cited 2016 Aug 18]. Available from: https://web.archive. org/web/20160818185430/http://www.who.int/mediacentre/news/releases/ 2016/nigeria-polio/en/.

56. Upfill-Brown AM, Lyons HM, Pate MA, Shuaib F, Baig S, Hu H, et al. Predictive spatial risk model of poliovirus to aid prioritization and hasten eradication in Nigeria. BMC Med [Internet]. 2014 [cited 2015 Aug 20];12:92. Available from: http://www.biomedcentral.com/1741-7015/12/92.

\section{Submit your next manuscript to BioMed Central and we will help you at every step:}

- We accept pre-submission inquiries

- Our selector tool helps you to find the most relevant journal

- We provide round the clock customer support

- Convenient online submission

- Thorough peer review

- Inclusion in PubMed and all major indexing services

- Maximum visibility for your research

Submit your manuscript at www biomedcentral.com/submit

\section{() BioMed Central}

